\title{
Spinal reflex plasticity in response to alpine skiing in the elderly
}

\author{
B. Lauber' ${ }^{1}$, M. Keller ${ }^{2}$, A. Gollhofer ${ }^{1}$, E. Müller ${ }^{3,4}$, W. Taube ${ }^{2}$ \\ ${ }^{1}$ Department of Sport Science, University of Freiburg, Freiburg, Germany, ${ }^{2}$ Department of Medicine, Movement and Sport Science, \\ University of Fribourg, Fribourg, Switzerland, ${ }^{3}$ Department of Sport Science and Kinesiology, University of Salzburg, Salzburg, \\ Austria, ${ }^{4}$ Christian Doppler Laboratory "Biomechanics in Skiing," Salzburg, Austria \\ Corresponding author: Benedikt Lauber, Department of Sport Science, University of Freiburg, Schwarzwaldstr 175, 79117 \\ Freiburg, Germany.Tel: +49 761203 4559, Fax: +49 761203 4534, E-mail: benedikt.lauber@sport.uni-freiburg.de
}

\begin{abstract}
The present study was designed to assess the influence of 12 weeks (28.5 $\pm \mathbf{2 . 6}$ skiing days) of alpine skiing on spinal reflex plasticity, strength and postural control in senior citizens. Therefore, soleus H-reflexes and postural stability were measured during bipedal quiet and unstable stance in 22 (12 male and 10 female) elderly subjects aged $66.6 \pm 1$ years. Furthermore, the maximal isometric force was determined in a leg press. The results showed an increased H-reflex excitability after the training $(+39 \% ; P<0.05)$ while no changes occurred in the background EMG. The postural sway
\end{abstract}

decreased after training $(-6.6 \mathrm{~cm} ; P \leq 0.05)$ and the maximal force increased $(+16.1 \% ; P<0.05)$. No adaptations in any parameter could be observed in the control group. The present study demonstrated that skiing training was effective to alter the spinal reflex activity in elderly individuals. The increased H-reflexes correspond to adaptations known from strength training in young subjects. It may be assumed that alpine skiing induced a functional adaptation in that subjects have learned to integrate Ia afferent feedback more efficiently to ensure adequate motoneuron output.
There is a rapid increase in the number of senior citizens in industrialized countries. Therefore, seniors represent a growing part of nation's population and their health and training status is consequently becoming of increasing interest. In the literature, the benefits of regular sportive activities on diseases like obesity or hypertension and on physical and mental well-being are very well documented (Nelson et al., 2007; Iwamoto et al., 2009). Furthermore, although the central nervous system of elderly individuals seems not to be as adaptable as that of young subjects (Scaglioni et al., 2002), several studies have shown that training induces neural plasticity in this population (Chalmers \& Knutzen, 2002; Mynark \& Koceja, 2002; Kamen \& Knight, 2004). So far, the influence of alpine skiing on adaptations of the neuromuscular system of elderly individuals is unknown. As skiing requires well-established postural skills (Müller, 2009; Noé et al., 2009) and strength capabilities (Neumayr et al., 2003), it was speculated that the central nervous system would adapt in order to accomplish the coordinative challenges. The adaptations on the spinal level going along with improvements in strength and postural control are well known: in longitudinal studies with young participants, diminished H-reflexes after several weeks of balance training were reported (for a review, see Taube et al., 2008a, b). The diminished excitability at the spinal level was speculated to reduce unwanted reflex initiated/(mediated) joint oscillations (Llewellyn et al., 1990; Aagaard et al., 2002). In contrast, strength training of the lower leg was shown to facilitate the reflex response (Aagaard et al., 2002; Lagerquist et al., 2006; Taube et al., 2007; Duclay et al., 2008). It was argued that the removal of inhibition might be beneficial to increase the power efficiency, which might be explained by an enhanced level of motoneuronal output (Sale et al., 1983; Voigt et al., 1998; Aagaard et al., 2002).

Unfortunately, only a few studies were conducted in the elderly to highlight this topic. Scaglioni et al. (2002) were the only ones to assess the H-reflex before and after 16 weeks of strength training in the elderly. However, they were not able to observe any training-induced changes of the reflex response. With respect to balance training, Mynark and Koceja (2002) demonstrated that elderly subjects were able to reduce their H-reflex amplitude in the same way as young subjects when peripheral nerve stimulation was used as a destabilizing stimulus. In this study, subjects were accustomed to the measuring device and the destabilizing stimuli on the first day. On two subsequent days, they were trained to control posture in the perturbation task. Thus, Mynark and Koceja demonstrated that elderly subjects could adapt their H-reflex response and their postural performance in response to a short-term training in a similar way as young subjects. However, 
when looking at instant adaptations of spinal reflexes in response to postural changes, it seems that elderly individuals's ability to modulate their H-reflex is less pronounced than that of young subjects (Chalmers \& Knutzen, 2002). Thus, although there might be differences in the adaptability of the spinal reflex circuits, these observations have demonstrated that elderly individuals do not completely lose the ability to modulate their reflex behavior task specifically. With respect to alpine skiing, it was hypothesized that both force and postural control would improve in response to this kind of intervention. Because of the welldocumented H-reflex adaptations in the elderly after balance training but not after strength training it was hypothesized that the 12 weeks of skiing would result in a reduction of the soleus H-reflex amplitude.

Post-activation (or homosynaptic) depression (PAD) might be another mechanism that could be affected by the skiing intervention. PAD reflects an inhibited Ia afferent transmitter release due to previous activation and lasts approximately $10 \mathrm{~s}$. The repetitive excitations cause a transmitter depletion within the presynaptic terminals and consequently a reduced postsynaptic excitation (Lev-Tov \& Pinco, 1992; Pinco \& Lev-Tov, 1993). Thus, PAD can be induced by a preceding conditioning stimulus, which activates the same afferents (Crone \& Nielsen, 1989; Lundbye-Jensen \& Nielsen, 2008). Based on the observation of a reduction in homosynaptic PAD after 2 weeks of immobilization (Lundbye-Jensen \& Nielsen, 2008) and by a study showing an increased level of PAD following stimulation of the tibial nerve and during mechanically evoked stretch reflexes of the soleus muscle (Grey et al., 2008), it was speculated that the increase in activity due to the skiing intervention may have the reverse effect, i.e. result in an increased level of PAD.

\section{Methods}

\section{Subjects}

For a general overview of the participants, please see Müller et al. (2010). Thirteen subjects were part of the intervention group (seven men and six women; $66.8 \pm 2$ years; $78.5 \pm 13.5 \mathrm{~kg} ; 168 \pm 7 \mathrm{~cm})$ and 10 subjects were part of the control group (five men and five women; $67.5 \pm 4$ years; $77.4 \pm 14.5 \mathrm{~kg} ; 169 \pm 8 \mathrm{~cm})$. The small number of subjects resulted from the circumstance that it was not possible to induce $\mathrm{H}$-reflexes in all subjects who took part in the study.

\section{Training}

The training included 12 weeks with a total number of $28.5 \pm 2.6$ skiing days, with an average of $3 \mathrm{~h} 27 \pm 14 \mathrm{~min}$ of skiing per skiing day (for more details regarding the skiing intervention, see Müller et al. 2010).

\section{Procedures}

After the preparation of the skin with sand paper and disinfectant spray, subjects were asked to rest on a massage couch with their face downwards. After finding the best spot for eliciting the H-reflex, an $H / M$ recruitment curve was obtained. A total number of 30 stimuli with an interstimulus interval of $4 \mathrm{~s}$ were applied while constantly increasing the intensity until $M_{\max }$ was obtained (in line with Aagaard et al., 2002; Zehr, 2002). This procedure was repeated while the subjects were standing in an upright position on both legs and while balancing on a spinning top (unstable condition) with their arms held akimbo. A spinning top is built of a circular, flat board mounted on top of a half-round ball mounted in the center of the board. Postural sway was measured for $30 \mathrm{~s}$ without peripheral nerve stimulation. Finally, postural sway and isometric maximum strength of the leg extensor muscles were tested in a leg press.

\section{EMG recordings}

EMG recordings were obtained from the $\mathrm{m}$. tibialis anterior (TA), m. gastrocnemius medialis (GM) and the $\mathrm{m}$. soleus (SOL) of the right leg. After the preparation of the skin, bipolar surface electrodes (Blue sensor P, Ambu, Bad Nauheim, Germany) were attached to the skin with a $2 \mathrm{~cm}$ interelectrode distance. EMG recordings were amplified $(\times 1000)$, bandpass filtered $(10-1000 \mathrm{~Hz})$ and sampled at $4 \mathrm{kHz}$. All data were stored in a custom-built software (LabView Based, National Instruments, Austin, Texas, USA) for off-line analysis. Muscular activity of SOL, TA and GM activity was quantified by root mean square values. The time window of analyses was set as 1.5-3.5 s after each stimulus for the following reasons: (i) the influence of the electric stimulus should be excluded, (ii) it was considered to be of importance to assess the muscular activity during the same trial as the electrophysiological data and (iii) the time window should be long enough to reveal the possible changes in EMG activity. EMG recordings were obtained in the prone position, during bipedal stable and unstable stance, and during measurements of the postural sway. Muscular activity of the SOL was expressed to the corresponding $M_{\max }$ to ensure comparability of pre- and post-measurements.

\section{H-reflex recording}

H-reflexes were elicited (AS 100, Alea Solutions ${ }^{\circledR}$, Zurich, Switzerland) in the right SOL muscle by stimulating the posterior tibial nerve in the poplitea fossa. For this purpose, the cathode $(2 \mathrm{~cm}$ in diameter) was moved stepwise until the best position for inducing an H-reflex was found. The anode, a $10 \times 5 \mathrm{~cm}$ dispersal pad, was fixed right below the patella on the anterior aspect of the knee. It was ensured that the electrical stimulation did not activate the TA. Stimulation intensity was constantly increased to record $H / M$ recruitment curves. Because the tibial nerve contains both, sensory and motor axons, the stimulation of this nerve induces a reflectory response (H-reflex with a latency of approximately $35 \mathrm{~ms}$ ) and a direct muscle response (M-wave with a latency of $\sim 10 \mathrm{~ms}$ ). The H-reflex travels mainly on Ia-affarents to the $\alpha$-motoneuron and from there to the muscular junction. The M-wave travels from the point of stimulation via motor axons straight to the muscular junction. When stimulating with low intensities, a small H-reflex can be observed without the presence of the Mwave due to the larger fiber diameter of the sensory axons. A constant increase of the stimulus intensity also recruits the smaller motor axons, resulting in an M-wave. By successively increasing the intensity from low to high stimuli, the H-reflex recruitment curve can be obtained (Zehr, 2002). To counteract the problem of unequal EMG recording conditions before and after the training, maximal H-reflexes were expressed in relation to $M_{\max }\left(H_{\max } / M_{\max }\right.$ ratio). The presented training effects were 
obtained by comparing the $H_{\max } / M_{\max }$ ratios taken from the $\mathrm{H}$ reflex recruitment curve recorded in pre- and post-measurement (in line with Aagaard et al., 2002). H-reflexes and M-waves were expressed as peak-to-peak amplitudes. To calculate the $H_{\max } /$ $M_{\max }$ ratios, the maximal amplitudes of the H-reflex and the Mwave were determined in each condition. No significant changes were observed in PAD.

\section{PAD}

In the present study, PAD was tested by applying $\mathrm{H}$-reflexes with a certain size $\left(20 \%\right.$ of $\left.M_{\max }\right)$ with two different interstimulus intervals: in the first case, the interstimulus interval was set to $10 \mathrm{~s}$. As PAD is known to last only for around $10 \mathrm{~s}$ (Crone \& Nielsen, 1989; Grey et al., 2008), this condition served as a control. In the second condition, 10 soleus $\mathrm{H}$-reflexes were applied with an interstimulus interval of $1 \mathrm{~s}$. In both conditions, it was analyzed how the preceding stimulus/stimuli affected the subsequent ones. Thus, the first stimulus of the 10 responses, which could not be affected by PAD, was excluded and the remaining nine responses were averaged. The amount of PAD was quantified as the difference between the averaged reflexsizes measured at $10 \mathrm{~s}$ (control condition) and 1-s interstimulus intervals (see also Lundbye-Jensen \& Nielsen, 2008).

\section{Postural sway}

The postural sway in the normal upright stance was assessed in pre- and post-measurements using a force plate (GKS $1000^{\mathbb{R}}$, IMM Holding GmbH, Mittweida, Germany) with a sampling frequency of $40 \mathrm{~Hz}$. For the duration of $30 \mathrm{~s}$, subjects were instructed to stand as quiet as possible with slightly flexed knees and arms held akimbo. In total, 21 subjects from the intervention group (13 men and eight female; 67.1 \pm 2 years; $80.1 \pm 14.6 \mathrm{~kg} ; 171.1 \pm 9.0 \mathrm{~cm})$ and 19 subjects from the control group (10 men and nine women; $67.4 \pm 4$ years; $72.0 \pm 12 \mathrm{~kg}$; $170.2 \pm 8.0 \mathrm{~cm})$ participated in this experiment. For each subject, the postural sway was evaluated in a normal upright bipedal stance. Therefore, the postural sway in the medio-lateral $(x)$ and the anterior-posterior direction $(y)$ was measured. The overall postural sway was calculated using the formula:

$$
\text { Sway }=\sum_{i: 1}^{n} \sqrt{\left(x_{1}-x_{0}\right)^{2}+\left(y_{1}-y_{0}\right)^{2}} \text {. }
$$

\section{Maximum isometric strength}

The maximal strength of both legs was tested in a leg press using an Isomed 2000 (R\&D, Hemau, Germany). The movement speed (concentric) was adjusted to a concentric speed of $100 \mathrm{~mm} / \mathrm{s}$ and an eccentric speed of $80 \mathrm{~mm} / \mathrm{s}$. The range of motion for the knees was from 100 to $150^{\circ}$ to ensure a similar knee angle as in alpine skiing and was controlled by a goniometer. The hip and the trunk were fixed. The backrest was adjusted to $75^{\circ}$ and the seat was kept horizontal. Before the testing started, subjects became familiar with the setup by performing 10 submaximal isokinetic contractions with a 60 -s rest in between. Thereafter, each subject performed three isometric contractions in the leg-press with maximal effort with a joint angle of $150^{\circ}$. During each trial, subjects were verbally encouraged to achieve a maximum force. The highest force peak $\left(F_{\text {peak }}\right)$ obtained in one of the three repetitions was taken as the maximal force parameter and was expressed relative to the body weight ( $F_{\text {max_r }}$ rel).

\section{Statistics}

Before a comparison of pre- and post-values was carried out, data were checked on normality using the Kolmogorov-Smirnov test.
Changes in $H_{\max } / M_{\max }$ ratios were analyzed using the repeated measures analysis of variance (ANOVA) procedure with factors condition (prone vs standing vs unstable stance), time (pre vs post) and group (intervention vs control) (3 [condition] $\times 2$ [time] $\times$ [group]). Changes in the postural sway were evaluated by repeated measures of ANOVA with factors $1 \times$ [postural sway] $\times 2$ [time] $\times 2$ [group]). Alterations in isometric $F_{\max }$ of both legs were captured by two-way repeated measures ANOVA with factors 2 [time] $\times 2$ [group].

If the results revealed significant $F$-values $(P<0.05)$, a Bonferroni corrected paired two-sided tests (Student's $t$-test) were performed in order to highlight differences in the preand post-values. Changes in the EMG patterns were measured by repeated ANOVA (3 [condition] $\times 2$ [time] $\times 3$ [muscle] $\times 2$ [group]).

SPSS $^{\circledR} 17$ software was used for all statistical analyses and results are presented as group mean values \pm SEM if not declared differently.

\section{Results}

$H_{\max } / M_{\max }$ ratios and EMG recordings

Reflex recordings of post-measurements revealed significant differences when compared with pre-measurement analyses with respect to the group (time group: $F=3.48 ; P<0.05)$. Thereby, the intervention group showed significant changes over time and condition (time $\times$ condition: $F=3.88 ; \quad P<0.05$ ), whereas the analyses of control group showed no effect $(F=1.2 ; P=0.33)$ (Fig. 1). In detail, for the training group, the $H_{\max } / M_{\max }$ ratios in the unstable

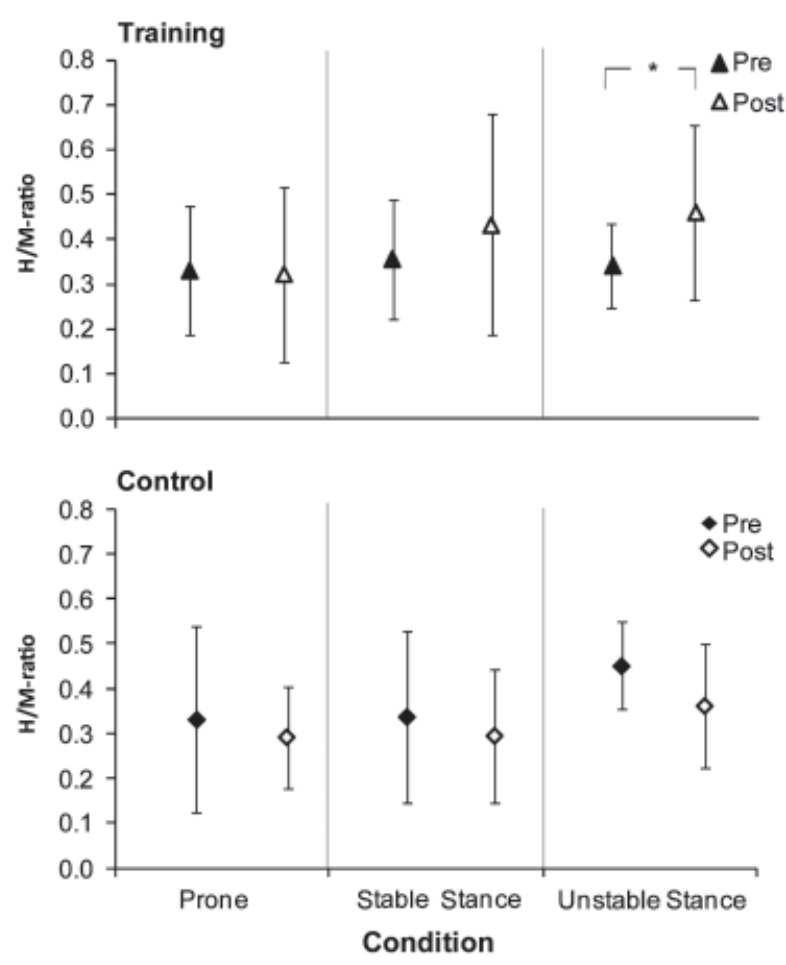

Fig. 1. $H / M$ ratios are displayed for the training (pre: $\mathbf{\Delta}$; post: $\triangle$ ) and the control group (pre: $\diamond$; post: $\diamond$ ). In all except the prone condition, an increase in reflex activity can be seen after the several weeks of skiing. No changes occurred in the control group. Error bars indicate SEM $\left({ }^{*} P<0.05\right)$. 


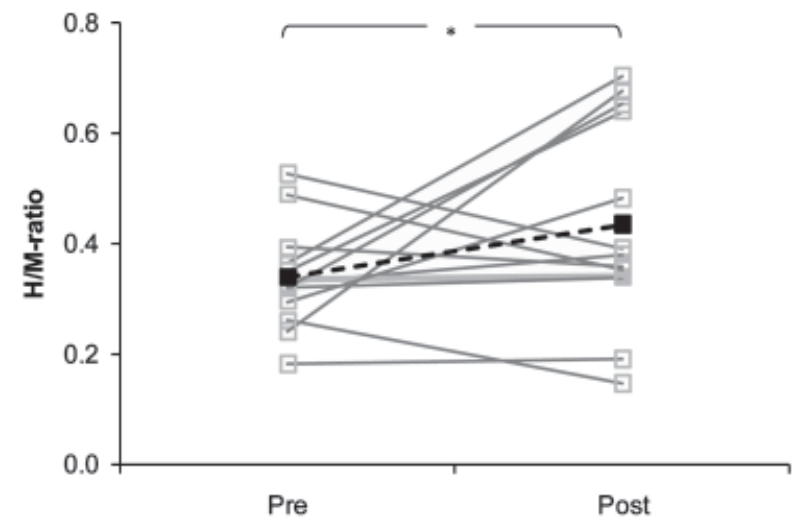

Fig. 2. $H / M$ ratios obtained when subjects were standing on a spinning top (unstable condition). A distinct overall increase in reflex activity became clear after the training intervention (dashed line) $(* P<0.05)$.

condition after training increased from 0.34 to 0.46 $(F=4.71 ; P=0.05)$ (Fig. 2). The muscular activity displayed no differences following the 12-week training period in any of the groups (non-normalized EMG: time $\times$ group $F=0.68 ; P=0.41$; normalized: time $\times$ group $F=3.19 ; P=0.1$. The EMG data of the soleus were additionally normalized to $M_{\max }$. However, as the results were similar to the nonnormalized data, we do not present them in the manuscript.

\section{Postural sway}

The postural sway in normal upright stance was decreased after the training regime $(24.6 \pm 2.0$ vs $18.0 \pm 2.0 \mathrm{~cm} ; F=3.16 ; P \leq 0.05$ ) (Fig. 3).

\section{Maximum isometric strength}

After the training, maximal strength of both legs was increased in both groups $(P<0.001)$. However, the increase in muscular strength was significantly greater in the intervention group (from 26.2 \pm 6.6 to $30.2 \pm 7.1 \mathrm{~N} / \mathrm{kg} ; 16.1 \pm 12.8 \%$ ) compared with the control subjects (from $27.0 \pm 6.5$ to $28.8 \pm$ $6.3 \mathrm{~N} / \mathrm{kg} ; 7.6 \pm 11.4 \%)(P<0.05)($ Fig. 4$)$.

\section{Discussion}

The aim of the present study was to evaluate the changes in spinal reflex activity after 12 weeks of alpine skiing in elderly individuals. The results demonstrate that alpine skiing is capable of modulating neuromuscular and biomechanical parameters like $H_{\max } / M_{\max }$ ratios, muscular strength and body sway in the elderly.

\section{H-reflex recruitment curves}

So far, there exists no study highlighting neurophysiological adaptations in response to alpine ski train-

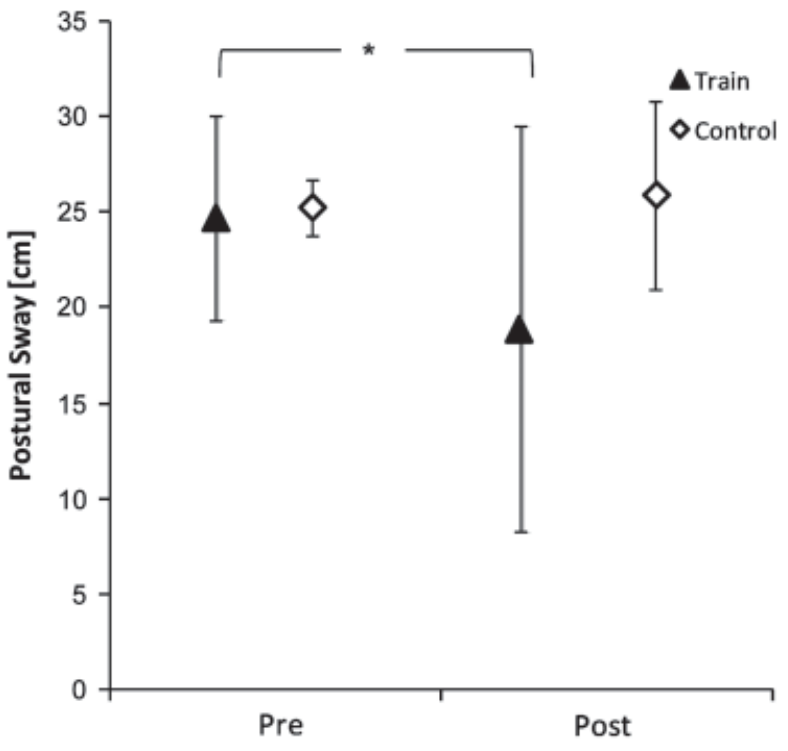

Fig. 3. Postural sway in the bipedal stance was decreased after the 12-weeks lasting training regime. The control group showed a slight increase after the training that was statistically not significant. Error bars indicate SEM $(* P<0.05)$.

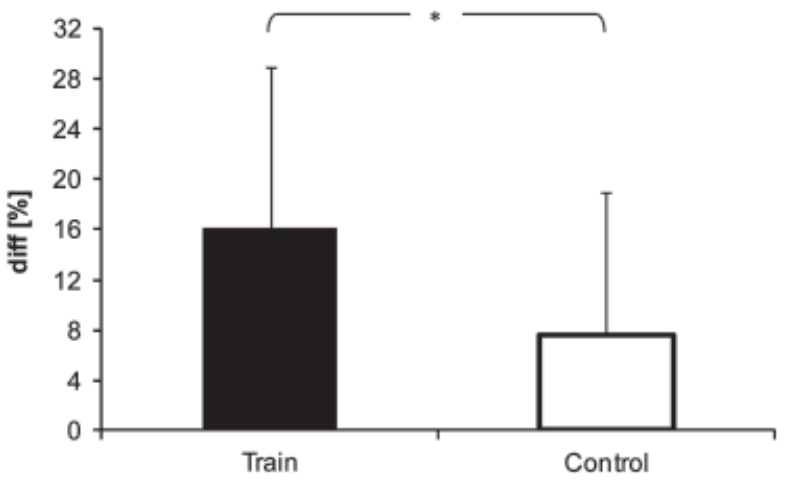

Fig. 4. Maximal isometric strength was increased after several weeks of alpine skiing in both groups. However, in comparison with the control group (white bar), a greater increase in $F_{\max }$ was present in the subjects who underwent the ski training (black bar). Error bars indicate SEM $(* P<0.05)$.

ing. In the present approach, it was therefore difficult to predict whether any adaptation of the soleus $\mathrm{H}$ reflex might be apparent and, if so, in which direction this adaptation may go. Previous studies have pointed out that the spinal reflex system adapts in a very specific manner depending on the exercise. Taube et al. (2007) reported decreased $H_{\max } / M_{\max }$ ratios after balance exercise but increased $H_{\max }$ $M_{\max }$ ratios after strength training. As skiing demands postural skills as well as sufficient and welltimed muscular force, it was speculated that skiing may improve balance control and strength capabilities in elderly subjects. However, with respect to the neural adaptations, it was speculated that the neural adaptations associated with balance training would be most prominent as the H-reflex of elderly 
individuals was shown to be reduced in response to balance exercises (Mynark \& Koceja, 2002) but remained unchanged following strength training (Scaglioni et al., 2002). Surprisingly, the present results revealed a different picture. Although both balance skills and strength capabilities were improved, there was a clear increase in the H-reflex amplitude after skiing training. The increase in $\mathrm{H}$ reflex excitability may suggest an increase in $\alpha$ motoneuron output. In analogy to strength training, it might be proposed that the facilitation of spinal reflex circuits after skiing helps the subjects to produce force in a more powerful way (Aagaard et al., 2002; Taube et al., 2007). Thus, counterproductive inhibitory mechanisms reducing the excitatory drive may be restrained in order to make use of the afferent feedback in a more efficient way (Earles et al., 2002). As the background EMG activity remained unchanged in all conditions (prone, stable and unstable stance) following the 12 weeks of alpine skiing, postsynaptic mechanisms like changes in reciprocal inhibition seem to be very unlikely. One could argue that the repetitive wearing of ski-boots flexing the knee approximately $100^{\circ}$ during skiing (Berg \& Eiken, 1999) may have shifted the overall body position in the anterior direction and therefore caused lengthening of the triceps surae complex, which is known to facilitate the soleus H-reflex (Nordlund et al., 2002). However, this seems to be very unlikely because it was shown that even highly experienced alpine-skiers who wear their ski-boots quite often do not alter their postural strategies when wearing no ski-boots (Noé \& Paillard, 2005; Noé et al., 2009).

Initially, we assumed that PAD would increase in response to the skiing activity as it was previously shown that immobilization resulted in reduced PAD (Lundbye-Jensen \& Nielsen, 2008) and skillful activity (cycle training) in an enhanced PAD (Meunier et al., 2007). The point that no changes in PAD were observed in this study might be related to the different training program or the different age group in our study compared with the previous studies. A change in PAD could have also influenced the Hreflex size during the recording of $H / M$-recruitment curves due to the relatively short interstimulus intervals (4s). However, as we could not find changes in PAD while testing with an interstimulus interval of $1 \mathrm{~s}$, it is unlikely that this mechanism influenced the increase in the H-reflex response. Based on our observations, it seems therefore more likely that a decrease in presynaptic inhibition (PSI) of Iaafferents may have accounted for the increased $H_{\max } / M_{\max }$ ratios. Former studies speculated that PSI of Ia-afferents has a strong influence on spinal reflex behavior during movements like walking and running (Faist et al., 1996; Hodapp et al., 2007) and that PSI can probably be modulated by training (Aagaard et al., 2002). Based on those previous findings, it might be speculated that the increased $H_{\max } / M_{\max }$ ratios following the 12 weeks of alpine skiing are the result of a reduced PSI.

\section{Similarities between skiing and strength training}

Based on the neural adaptations presented in this study, it can be assumed that skiing training acts in a similar way as strength training. The $F_{\max }$ measurements confirm this assumption and demonstrate significantly increased force levels after the skiing intervention. This seems reasonable as skiing is characterized by constant alterations of concentric and eccentric contractions of the leg muscles as well as phases of prolonged co-contraction (Hintermeister et al., 1995). Dependent on the skiing discipline and the skiing intensity, variations in the contraction strength and contraction time occur. Previously, it was shown that during giant slalom and slalom, EMG activity in the calf muscles is very high (Berg et al., 1995; Hintermeister et al., 1995; Berg \& Eiken, 1999). Thus, it seems rational that skiing incorporates sufficient neuromuscular stimuli to ameliorate the force capabilities.

\section{Changes in postural control}

The influence of alpine skiing on balance parameters is poorly understood. To our knowledge, the present intervention is the first one to directly indicate that balance control can be improved in response to skiing training in elderly individuals. However, it has to be admitted that the reduced postural sway does not consequently indicate enhanced postural control. Studies on patients with Parkinson's disease also reported decreased body sway when subjects were investigated in an upright stance on a stable surface (Horak et al., 1992; Schieppati et al., 1994; Smithson et al., 1998). However, when postural demands were increased and the patients had to perform a single-leg stance, significant problems in maintaining balance control were observed. The present study did not test postural stability in more challenging situations and therefore cannot make any assumptions about the functionality of the observed adaptations in postural sway displacement. However, it seems rational that skiing can improve postural control as it demands to keep the center of mass over the support surface (skis) despite constant variations in terms of loading, contraction velocity and contraction time. The subjects who participated in this study were divided into groups according to their skiing abilities but were allowed to ski at their own speed within their group.

We were not able to detect any correlation between changes in postural control and maximal leg 
strength. One reason might be that improvements in balance control were demonstrated to be more closely related to improvements in power than to muscular strength (Orr et al., 2006). Unfortunately, no power measurements were performed in the present study to clarify this point.

\section{H-reflex and postural sway}

The present study showed that the postural sway was reduced despite increases in the H-reflex amplitude. This is highly interesting as all previous balance training studies reported decreases or non-adaptations in the H-reflex going along with improved postural control (Chalmers \& Knutzen, 2002; Mynark \& Koceja, 2002; Taube et al., 2008a). Similarly, H-reflex excitability was lower when subjects were tested in more challenging situations like tandem stance (Chalmers \& Knutzen, 2002), stance on elevated platforms or when standing with the eyes closed (Hoffman \& Koceja, 1995; Earles et al., 2000). In contrast, when the demands were lowered by utilizing additional feedback by means of a laser pointer (Taube et al., 2008b) or additional mechanical support (Tokuno et al., 2009), the H-reflex amplitudes were increased. Thus, the central nervous system seems to respond instantly to the demands of the postural task. As the demands of the postural test situation were not seriously challenging and did not change in the present study but subjects showed enhanced H-reflexes in the post-measurement, it might be speculated that they perceived the task to be less challenging after the skiing intervention. As with maximal strength of the leg muscles, we were not able to find significant correlations between the increased H-reflex excitability and a reduced postural sway following the alpine skiing. One explanation could be that it seems that the control of human standing is at least partly related to changes in the excitability of the motor cortex (Tokuno et al., 2009). In this context, Taube et al. (2007) showed that balance-training induced adaptations of the cortical excitability were correlated to changes in stance stability but not to changes of the H-reflex.

\section{Conclusion and perspectives}

In summary, this study showed that several weeks of alpine skiing increased H-reflex excitability and muscular strength and decreased the amount of postural sway. The neural adaptations at the spinal level are similar to those observed in young subjects after strength training and may serve to enhance power by disinhibiting motoneuron output.

Key words: peripheral nerve stimulation, balance, alpine skiing, maximal isometric strength, seniors.

Conflicts of interest: The authors have no potential conflicts of interest.

\section{References}

Aagaard P, Simonsen EB, Andersen JL, Magnusson P, Dyhre-Poulsen P. Neural adaptation to resistance training: changes in evoked $\mathrm{V}$-wave and H-reflex responses. J Appl Physiol 2002: 92: 2309-2318.

Berg HE, Eiken O. Muscle control in elite alpine skiing. Med Sci Sports Exerc 1999: 31: 1065-1067.

Berg HE, Eiken O, Tesch PA. Involvement of eccentric muscle actions in giant slalom racing. Med Sci Sports Exerc 1995: 27: 1666-1670.

Chalmers GR, Knutzen KM. Soleus Hreflex gain in healthy elderly and young adults when lying, standing, and balancing. J Gerontol A Biol Sci Med Sci 2002: 57: 321-329.

Crone C, Nielsen J. Methodological implications of the post activation depression of the soleus H-reflex in man. Exp Brain Res 1989: 78(1): 28-32.

Duclay J, Martin A, Robbe A, Pousson M. Spinal reflex plasticity during maximal dynamic contractions after eccentric training. Med Sci Sports Exerc 2008: 40: 722-734.

Earles DR, Dierking JT, Robertson CT, Koceja DM. Pre- and post-synaptic control of motoneuron excitability in athletes. Med Sci Sports Exerc 2002: 34: 1766-1772.

Earles DR, Koceja DM, Shively CW. Environmental changes in soleus H-reflex excitability in young and elderly subjects. Int J Neurosci 2000: 105: 1-13.

Faist M, Dietz V, Pierrot-Deseilligny E. Modulation, probably presynaptic in origin, of monosynaptic Ia excitation during human gait. Exp Brain Res 1996: 109: 441-449.

Grey MJ, Klinge K, Crone C, Lorentzen J, Biering-Sorensen F, Ravnborg M, Nielsen JB. Post-activation depression of soleus stretch reflexes in healthy and spastic humans. Exp Brain Res 2008: 185: 189-197.

Hintermeister RA, O’Connor DD, Dillman CJ, Suplizio CL, Lange GW,
Steadman JR. Muscle activity in slalom and giant slalom skiing. Med Sci Sports Exerc 1995: 27: 315-322.

Hodapp M, Klisch C, Berger W, Mall V, Faist M. Modulation of soleus Hreflexes during gait in healthy children. Exp Brain Res 2007: 178: 252-260.

Hoffman MA, Koceja DM. The effects of vision and task complexity on Hoffmann reflex gain. Brain Res 1995: 700: 303-307.

Horak FB, Nutt JG, Nashner LM. Postural inflexibility in parkinsonian subjects. J Neurol Sci 1992: 111: 46-58.

Iwamoto J, Suzuki H, Tanaka K, Kumakubo T, Hirabayashi $\mathrm{H}$, Miyazaki Y, Sato Y, Takeda T, Matsumoto H. Preventative effect of exercise against falls in the elderly. A randomized controlled trial. Osteoporo Int 2009: 20: 1233-1240.

Kamen G, Knight CA. Training-related adaptations in motor unit discharge rate in young and older adults. 
J Gerontol Ser A, Biol Sci Med Sci 2004: 59: 1334-1338.

Lagerquist O, Zehr EP, Docherty D. Increased spinal reflex excitability is not associated with neural plasticity underlying the cross-education effect. J Appl Physiol 2006: 100: 83-90.

Lev-Tov A, Pinco M. In vitro studies of prolonged synaptic depression in the neonatal rat spinal cord. J Physiol 1992: 447: 149-169.

Llewellyn M, Yang JF, Prochazka A. Human H-reflexes are smaller in difficult beam walking than in normal treadmill walking. Exp Brain Res 1990: 83: 22-28.

Lundbye-Jensen J, Nielsen JB. Immobilization induces changes in presynaptic control of group Ia afferents in healthy humans. J Physiol 2008: 586: 4121-4135.

Meunier S, Kwon J, Russmann H, Ravindran S, Mazzocchio R, Cohen L. Spinal use-dependent plasticity of synaptic transmission in humans after a single cycling session. J Physiol 2007: 579: 375-388.

Müller E, Gimpel M, Poetzelsberger B, Finkenzeller T, Scheiber P. SASES Salzburg Skiing for the Elderly Study: study design and intervention. Health benefit of alpine skiing for the elderly. Scand J Med Sci Sports 2011: 21(Suppl. 1): $1-8$.

Müller W. Determinants of ski-jump performance and implications for health, safety and fairness. Sports Med 2009: 39: 85-106.

Mynark RG, Koceja DM. Down training of the elderly soleus $\mathrm{H}$ reflex with the use of a spinally induced balance perturbation. J Appl Physiol 2002: 93: 127-133.

Nelson ME, Rejeski WJ, Blair SN, Duncan PW, Judge JO, King AC,
Macera CA, Castaneda-Sceppa C. Physical activity and public health in older adults: recommendation from the American College of Sports Medicine and the American Heart Association. Med Sci Sports Exerc 2007: 39: 1435-1445.

Neumayr G, Hoertnagl H, Pfister R, Koller A, Eibl G, Raas E. Physical and physiological factors associated with success in professional alpine skiing. Int J Sports Med 2003: 24: 571-575.

Noé F, Amarantini D, Paillard T. How experienced alpine-skiers cope with restrictions of ankle degrees-offreedom when wearing ski-boots in postural exercises. J Electromyogr Kinesiol 2009: 19: 341-346.

Noé F, Paillard T. Is postural control affected by expertise in alpine skiing? Br J Sports Med 2005: 39: 835-837.

Nordlund MM, Thorstensson A, Cresswell AG. Variations in the soleus $\mathrm{H}$-reflex as a function of activation during controlled lengthening and shortening actions. Brain Res 2002: 952: 301-307.

Orr R, Vos NJ, de Singh NA, Ross DA, Stavrinos TM, Fiatarone-Singh MA. Power training improves balance in healthy older adults. J Gerontol A Biol Sci Med Sci 2006: 61: 78-85.

Pinco M, Lev-Tov A. Modulation of monosynaptic excitation in the neonatal rat spinal cord. $\mathbf{J}$ Neurophysiol 1993: 70: 1151-1158.

Sale DG, MacDougall JD, Upton AR, McComas AJ. Effect of strength training upon motoneuron excitability in man. Med Sci Sports Exerc 1983: 15(1): 57-62.

Scaglioni G, Ferri A, Minetti AE, Martin A, van Hoecke J, Capodaglio P, Sartorio A, Narici MV. Plantar flexor activation capacity and $\mathrm{H}$ reflex in older adults: adaptations to strength training. J Appl Physiol 2002: 92: 2292 2302.

Schieppati M, Hugon M, Grasso M, Nardone A, Galante M. The limits of equilibrium in young and elderly normal subjects and in parkinsonians. Electroencephalogr Clin Neurophysiol 1994: 93: 286-298.

Smithson F, Morris ME, Iansek R. Performance on clinical tests of balance in Parkinson's disease. Phys Ther 1998: 78: 577-592.

Taube W, Gruber M, Gollhofer A. Spinal and supraspinal adaptations associated with balance training and their functional relevance. Acta Physiol 2008a: 193: 101-116.

Taube W, Kullmann N, Leukel C, Kurz O, Amtage F, Gollhofer A. Differential reflex adaptations following sensorimotor and strength training in young elite athletes. Int J Sports Med 2007: 28: 999-1005.

Taube W, Leukel C, Gollhofer A. Influence of enhanced visual feedback on postural control and spinal reflex modulation during stance. Exp Brain Res 2008b: 188: 353-361.

Tokuno CD, Taube W, Cresswell AG. An enhanced level of motor cortical excitability during the control of human standing. Acta Physiol 2009: 195: 385-395.

Voigt M, Chelli F, Frigo C. Changes in the excitability of soleus muscle short latency stretch reflexes during human hopping after 4 weeks of hopping training. Eur J Appl Physiol Occup Physiol 1998: 78: 522-532.

Zehr EP. Considerations for use of the Hoffmann reflex in exercise studies. Eur J Appl Physiol 2002: 86: 455-468. 